\title{
Abstração Refletida sobre o Processo de Gênese Instrumental em Geometria Dinâmica: Restrições, Desafios, Novas Possibilidades de Aprendizagem
}

\author{
Margarete Farias Medeiros - IFC - CAS/PPGIE - UFRGS - \\ margarete.medeiros@ifc.edu.br \\ Marcus Basso - PPGEMAT/IME/UFRGS - PPGIE - mbasso@ufrgs.br
}

\section{Resumo:}

O presente artigo expõe um recorte de uma pesquisa em andamento, de natureza qualitativa e cunho exploratório, no ambiente de geometria dinâmica GeoGebra 3D. Utilizando determinadas restrições que o software apresenta, desafios que nele podem ser criados e possibilidades de ensino e aprendizagem da matemática escolar. A pesquisa, fundamentada no mecanismo da abstração reflexionante e na teoria da gênese instrumental, foi realizada a partir de observações sobre os comportamentos de estudantes em formação inicial de professor de matemática durante a execução de tarefas sobre conceitos de geometria espacial utilizando tal tecnologia. A partir da análise dos dados foi possível concluir que existem fortes indícios de que os sujeitos supracitados submetidos às restrições do ambiente: ultrapassaram os desafios propostos; exploraram as novas possibilidades oportunizadas pelo ambiente dinâmico; e apresentaram tal comportamento indicando a abstração refletida sobre o seu processo de gênese instrumental.

Palavras-Chave: geometria dinâmica, abstração refletida, gênese instrumental, formação inicial docente.

\section{Abstractions reflected on the process of instrumental Genesis in dynamic Geometry: Constraints, challenges, new learning possibilities}

\begin{abstract}
:
This paper presents a study in progress, of qualitative and exploratory nature, in the GeoGebra 3D dynamic geometry environment. Using certain restrictions that the software presents, challenges that can be created in it and possibilities of teaching and learning of school mathematics. The research, based on the mechanism of reflexionant abstraction and the theory of instrumental genesis, was made from observations on the behaviors of students in initial training of mathematics teachers during the execution of spatial geometry concepts tasks using such technology. From the data analysis it was possible to conclude that there are strong indications that the aforementioned subjects submitted to the constraints of the environment: exceeded the proposed challenges; explored the new possibilities that were granted by the dynamic environment; and they presented such behavior indicating the reflected abstraction about their instrumental genesis process.
\end{abstract}

Keywords: Dynamic geometry, reflected abstraction, instrumental genesis, initial teacher training.

\section{Introdução}

Em tempos digitais, pesquisadores apontam que a formação docente deve acompanhar os avanços tecnológicos, permitindo ao futuro professor condições V. $17 \mathrm{~N}^{\circ}$ 3, dezembro, 2019 DOI: RENOTE 
favoráveis à inserção da TD (Tecnologia Digital) na Educação Matemática (ROCK, 2017; NOTARE E BASSO, 2017). Nosso pensar sobre isso parte do fato de que este futuro professor conduzirá os processos de ensino e aprendizagem da matemática escolar e que seu processo de gênese instrumental será fundamental nesta tarefa. "Não basta introduzir o computador, ou os softwares nos ambientes escolares, é necessária a transformação do artefato em instrumento por parte do professor, ou seja, que aconteça a gênese instrumental." (MEDEIROS E BASSO, 2018). Consideramos a TD como ferramenta essencial para a aprendizagem em matemática, fundamentando-nos nas palavras de Trouche (2018, p.1, Trad. nossa), o qual enfatiza que "(...) a importância das ferramentas na atividade matemática tem sido amplamente reconhecida".

Nesta direção buscamos conduzir uma pesquisa que tratasse do estudo do mecanismo da abstração reflexionante sobre o processo de gênese instrumental de futuros professores de matemática por meio de um ambiente de geometria dinâmica, neste contexto, o GeoGebra 3D. O que ele (o software) pode oferecer para contribuir no processo de ensino e aprendizagem? Possui restrições? Permite criar desafios e, por consequência, oportunizar possibilidades de aprendizagem? A partir de quais características do software podemos inferir a apropriação tecnológica por parte do sujeito? De acordo com BRETSCHER (2009), a exploração da variação dinâmica para mostrar a invariância do objeto matemático construído é uma forma de reconhecer o potencial do software. $\mathrm{O}$ autor considera um aspecto fundamental, no apoio à gênese instrumental dos alunos, tornar explícitos os recursos do software.

\section{Quadro Teórico}

Fundamentando nosso estudo do processo de gênese instrumental, buscamos apoio nos recentes estudos de Trouche (2018) e seus colaboradores, além do mecanismo da abstração reflexionante de Piaget (1977/1995). Conduzimos uma conexão entre as duas teorias na qual consideramos que o estudante poderá ter uma abstração refletida sobre o seu processo de gênese instrumental, levando-o à incorporação do ambiente de geometria dinâmica a partir dos recursos do software. De acordo com Hegedus e Tall (2016), os recursos de ambientes dinâmicos de matemática oportunizam ao estudante: explorar, expressar, visualizar, calcular e interagir com objetos matemáticos.

Para apresentarmos esta conexão entre as duas teorias supracitadas, trataremos inicialmente de explicitar como entendemos o processo de gênese instrumental fundamentados em autores que o abordam.

O processo de gênese instrumental foi inserido por Rabardel (1995), definindo-o como uma transformação por parte do sujeito de um artefato em um instrumento ao realizar uma atividade, no entanto, ele não definiu artefatos e instrumentos como sendo sinônimos. $\mathrm{O}$ artefato foi classificado como algo material ou não, produzido pelo homem, como uma calculadora, um algoritmo, um software, dentre outros. Já instrumento foi definido como uma entidade composta por um artefato acrescido pelo sujeito de um esquema mental (VERGNAUD, 1996), sendo este esquema resultado da interação entre o sujeito e o artefato.

Nos desdobramentos de Jupri, Drijvers e Heuvel-Panhuizen (2016), o instrumento é visto como uma entidade mista formada de esquema, técnica, artefato e tarefa. $\mathrm{O}$ esquema vem de acordo com Vergnaud (1996), o qual fundamenta-se em Piaget, e a técnica em consonância com Artigue (2002). Segundo Drijvers (2012), as técnicas são consideradas como manifestações observáveis dos esquemas invisíveis quando o sujeito 
realiza uma tarefa, portanto elas podem ser observadas por meio do comportamento do sujeito ao utilizar o artefato.

Neste processo de gênese instrumental, Trouche (2015) esclarece que existem duas dimensões: a instrumentação orientada para o sujeito e a instrumentalização orientada para o artefato. "A instrumentação é a ação para dar a alguém um instrumento, ou a ação pela qual alguém adquire um instrumento, a fim de realizar uma determinada atividade." (TROUCHE, 2018, p.1 Trad nossa).

Rabardel (1995) estabeleceu uma tríade neste processo de gênese instrumental: sujeito, instrumento e objeto. Os desdobramentos desta teoria apontados por Maschietto e Trouche (2009) mostraram que este processo, que demanda tempo, é complexo, individual, ligado às potencialidades e restrições do artefato, às atividades do sujeito, seu conhecimento e método de trabalhos anteriores.

No estudo em questão, o sujeito é o professor de matemática em formação inicial; o artefato, o ambiente de geometria dinâmica GeoGebra 3D; os objetos são conceitos de geometria espacial: por exemplo, a construção de um applet que simule o enchimento de um reservatório em formato de cilindro circular. $\mathrm{E}$ o instrumento? $\mathrm{O}$ instrumento é o GeoGebra 3D acrescido dos esquemas (VERGNAUD, 1996) mentais que o sujeito criará para responder a uma tarefa no ambiente dinâmico a partir dos recursos que ele (o software) apresenta. Para isso o sujeito buscará em seus conhecimentos prévios, tanto dos conceitos geométricos quanto das explorações das ferramentas do software para completar a tarefa proposta.

Ao ser submetido a uma tarefa neste ambiente dinâmico, o sujeito se depara com restrições, potencialidades do software e desafios a serem superados, e, a partir dos seus comportamentos, frente à tarefa, observamos o mecanismo da abstração reflexionante (PIAGET, 1977/1995) com tomada de consciência (abstração refletida) sobre o seu processo de gênese instrumental.

Piaget (1977/1995) apresenta diferentes tipos de abstração, sendo que na reflexionante o sujeito retira qualidades não do objeto, mas da sua própria ação sobre o objeto. Consideramos que o sujeito terá uma abstração refletida, reflexionante com tomada de consciência, quando ao coordenar suas ações sobre o objeto, retirar qualidades destas e generalizar para o infinito. Neste contexto as ações estão relacionadas com o processo de gênese instrumental (a transformação do artefato em instrumento pelo sujeito ao realizar tarefas no ambiente 3D). Ao realizar tarefas o sujeito tomará consciência, por ocasião de uma abstração refletida, de sua apropriação em relação às características do ambiente, podendo se utilizar delas para as infinitas construções. O mecanismo da abstração reflexionante é uma atividade mental e não pode ser observada, ela é interna, podemos apenas inferir a partir da observação do comportamento do sujeito (BECKER, 2014).

O processo de abstração reflexionante é composto por reflexionamentos e reflexões. De acordo com Becker (2012a, p.95), o reflexionamento consiste na "(...) projeção sobre um patamar superior daquilo que foi tirado do patamar inferior (...) e a reflexão é entendida como um "ato mental de reconstrução e reorganização sobre o patamar superior daquilo que foi assim transferido do inferior" (PIAGET, 1977/1995, p. 275). É um processo contínuo e ininterrupto formando novos patamares, um processo em espiral.

Para apresentar esta conexão entre o mecanismo da abstração reflexionante (abstração refletida, PIAGET, 1977/1995) com o processo de gênese instrumental, elaboramos um modelo (Figura 1) fundamentado na tríade de Rabardel (1995) e em 
Maschietto e Trouche (2009), os quais apresentam a interação entre sujeito, artefato e objeto; no decorrer do tempo, destacando os componentes da gênese instrumental: a instrumentação e instrumentalização.

Ao nosso ver, é a partir da abstração refletida sobre o seu processo de gênese instrumental que o sujeito tomará consciência de sua própria instrumentação em relação ao GeoGebra 3D e seus recursos por ocasião das apropriações tecnológicas. Ponderamos que diante desse fato o professor de matemática em formação inicial terá condições para inserir tal TD na sua prática docente. Consideramos a tomada de consciência em relação às características do ambiente, às restrições e potencialidades: a dependência funcional geométrica, a estabilidade do objeto construído sob a ação de movimentos, e a limitação do ambiente lápis e papel em relação ao dinâmico.

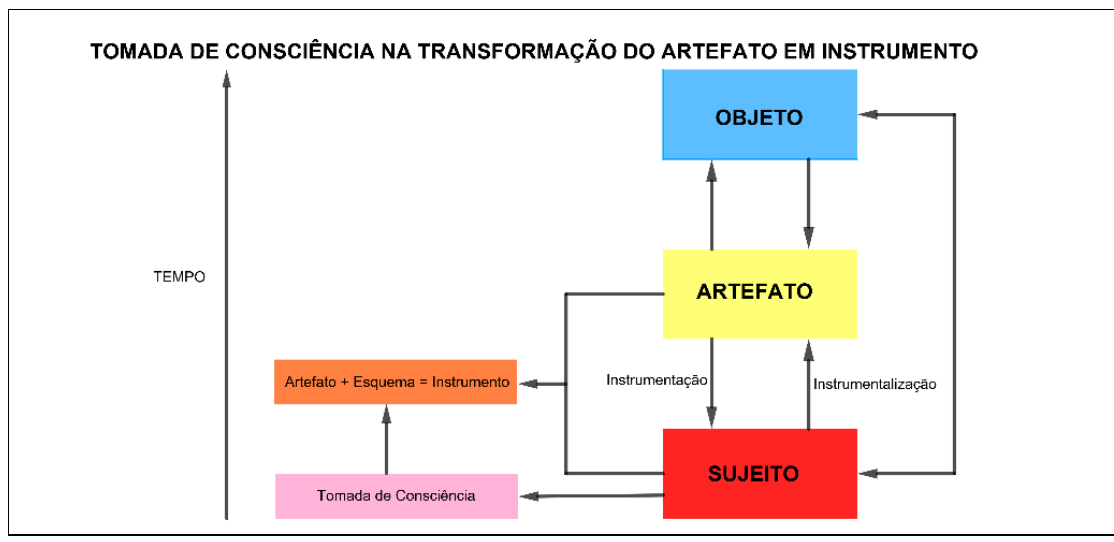

Figura 1: Abstração Refletida sobre o processo de gênese instrumental Fonte: Os autores

No que segue apresentamos um recorte da pesquisa em andamento com resultados das análises do comportamento de dois sujeitos submetidos a uma tarefa neste ambiente 3D. Embora tenhamos resultados parciais, observamos comportamentos indicando a abstração refletida sobre o seu processo de gênese instrumental.

\section{Resultados e Discussões}

A presente pesquisa inicialmente foi conduzida por um pesquisador analisando o comportamento de dez estudantes do curso de licenciatura em matemática, no entanto, ao final do projeto foram selecionados apenas cinco estudantes, utilizando-se como critério de seleção aqueles que participaram em todas as atividades.

Como instrumentos de produção de dados foram realizadas: 25 tarefas no GeoGebra 3D; escritas sobre as dificuldades ao realizar as tarefas, tanto em relação aos conceitos matemáticos quanto à utilização do software; observação dos comportamentos dos estudantes nas dez oficinas filmadas: cinco de ação instrumentada (3h30min cada executadas na disciplina de Tecnologias Digitais no Ensino de Matemática) e cinco de simulação de atividade profissional (5 turnos de $3 \mathrm{~h}$ cada); respostas às entrevistas (uma escrita via Google docs e uma presencial inspirada no método clínico).

Neste artigo expomos as análises em relação a dois estudantes, os quais apresentaram indícios de terem tido a abstração refletida sobre o seu processo de gênese instrumental na mesma oficina instrumentada. As demais análises dos outros estudantes estão sendo tratadas na tese em estudo buscando evidências a fim de comprovar o modelo proposto. 
No que segue apresentamos as análises: da produção de uma tarefa (construção de applet simulando o enchimento de um reservatório no formato de cilindro circular, constituindo-se de uma modelagem geométrica); dos comportamentos dos dois estudantes na quarta oficina instrumentada (os quais mostraram indícios da abstração refletida sobre seu processo de gênese instrumental).

\subsection{A quinta tarefa}

Cada oficina instrumentada, com exceção da primeira oficina, era composta de cinco tarefas para serem realizadas no GeoGebra 3D. As construções deveriam obedecer aos princípios da geometria dinâmica, além de que os estudantes não poderiam utilizarse de ferramentas automáticas e deveriam se apoiar em relações matemáticas. A cada oficina o nível de dificuldade da tarefa aumentava, tanto em relação aos conceitos geométricos quanto ao rol de ferramentas que deveriam utilizar, ou seja, os desafios iam aumentando. A análise da produção da tarefa foi realizada a partir do protocolo de construções fornecido pelo GeoGebra 3D, o qual permite verificar os passos seguidos pelo estudante ao realizar a atividade. Os dois estudantes, Renata e Yago, foram apresentados com nomes fictícios preservando suas identidades.

A tarefa escolhida foi a de número cinco, a última construída na quarta oficina instrumentada: Construa um applet que simule o enchimento de um reservatório no formato de cilindro circular. Esta tarefa foi fundamentada na ideia de Meier e Gravina (2012) sobre a criação de uma modelagem geométrica, mostrando o mecanismo em funcionamento no qual as formas geométricas deveriam estar em movimento. "Uma modelagem geométrica é uma representação, na linguagem da matemática, de um mecanismo no qual as formas geométricas estão em movimento" (MEIER E GRAVINA, 2012).

No arquivo de Renata (Figura 2) temos uma construção estável e dinâmica preservando as propriedades iniciais dos objetos inseridos. Na figura 2, temos destacado o protocolo de construções apresentando as etapas de construção do applet.

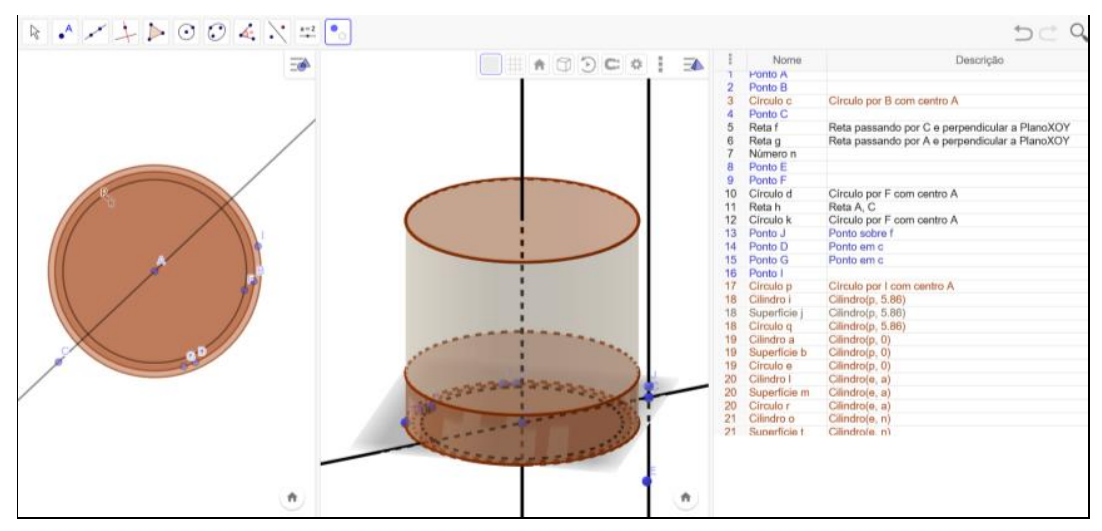

Figura 2: Construção do applet do cilindro

Fonte: Os autores

Na Figura 3, temos a tarefa representada por Renata com diversos objetos escondidos, deixando à mostra somente o que representaria o enchimento de um reservatório cilíndrico. Ao movimentarmos o controle deslizante $n$ temos a variação da altura do cilindro $o$, representando o enchimento de um reservatório conforme solicitado na tarefa por meio de uma representação dinâmica. 


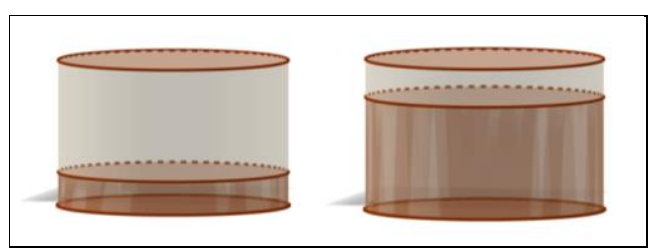

Figura 3: Representação dinâmica no ambiente Fonte: Os autores

No arquivo de Yago (Figura 4) verificamos os objetos auxiliares para a construção bem como o protocolo de construções. Sua construção também manteve os princípios da geometria dinâmica, a estabilidade sob ação de movimentos.

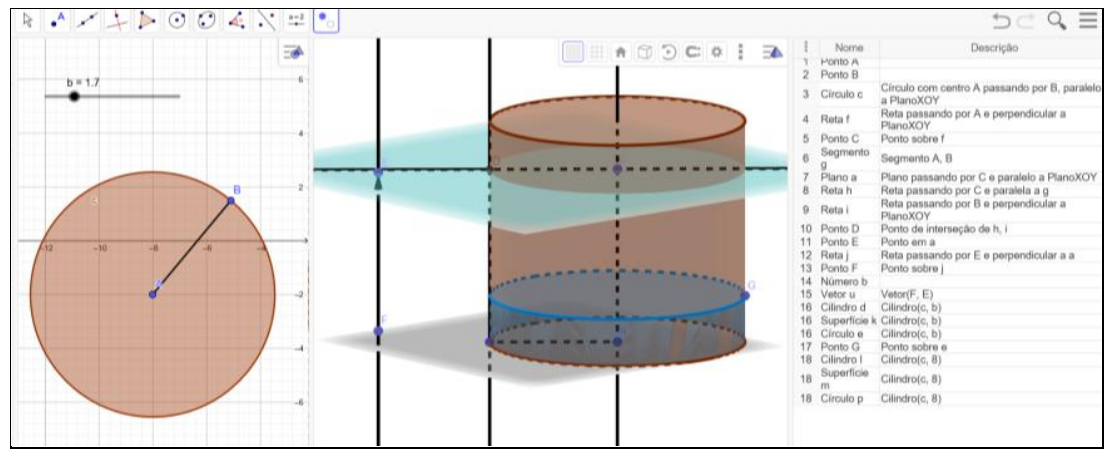

Figura 4: Construção do applet do cilindro Fonte: Os autores

Com a animação do número $b$ (controle deslizante) (Figura 3), a construção de Yago representou o enchimento de um reservatório cilíndrico: considerou o cilindro $l$ como sendo o recipiente (altura fixa em 8) e o cilindro $d$ (com altura variando de 0 a 8 ) representando o enchimento do cilindro $l$ durante um intervalo de tempo $t$. Na Figura 4 apresentamos três imagens mostrando a dinamicidade do applet construído no ambiente $3 \mathrm{D}$.

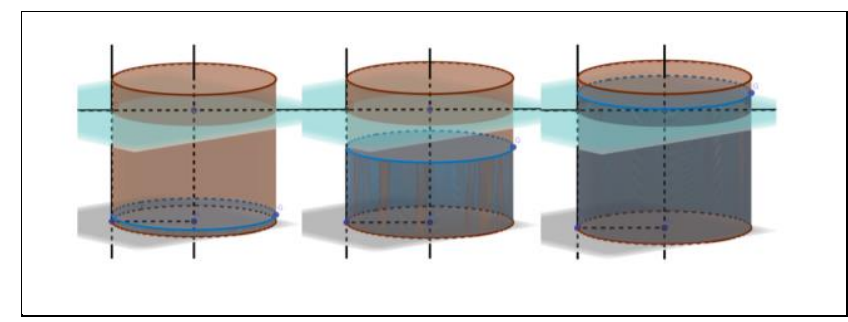

Figura 4: Representação dinâmica no ambiente Fonte: Os autores

Ao analisarmos as produções dos estudantes, observamos que ambos conseguiram completar a tarefa corretamente, produzindo um applet de acordo com os princípios da geometria dinâmica, evidenciando a apropriação das características do ambiente.

\subsection{O comportamento dos estudantes}

Observamos o comportamento in loco, além da análise do vídeo da quarta oficina instrumentada. Do vídeo da oficina extraímos as falas dos estudantes que indicam que pode ter ocorrido a abstração refletida sobre o seu processo de gênese instrumental. No que segue apresentamos estas falas.

O estudante Yago, depois de fazer explicações às duas colegas, chamou-nos para se expressar: 
Yago: "Parece que só vai aumentando o nível das coisas. Por exemplo: eu, se fosse no começo das aulas, ... eu teria muitas dificuldades de fazer pelas propriedades".

A partir de sua fala, nós confirmamos o que ele havia percebido, que as atividades foram organizadas de forma que fosse aumentando o nível de dificuldade. Nesta direção ele percebeu o acréscimo do nível de dificuldade, além de fazer uma análise de seu comportamento em relação às tarefas. Conjecturamos que ele tenha tido uma abstração refletida sobre o seu processo de gênese instrumental, quando ao final da discussão ele afirmou:

Yago: "Hoje é mais fácil".

No que tange à estudante Renata, sobre a qual nós julgamos importante, sua apropriação do GeoGebra 3D, dando indícios de ter consciência sobre o seu processo de gênese instrumental pessoal por meio de sua expressão facial de deslumbramento mostrando esta consciência do seu processo:

Renata: "Nossa professora como a gente muda. Como a gente muda 'né'?"

Neste instante ela olhou para o estudante Yago e acrescentou:

Renata: "Olha o nível que nós estamos fazendo as coisas?

E Yago respondeu:

Yago: "Foi isso que eu falei para ela".

Ele estava se referindo à conversa que tivemos sobre essa percepção de que o 'hoje' estava mais fácil de responder à tarefa.

Renata continuou:

Renata: "Eu não sabia fazer nada (...)".

Entramos na discussão dos dois sobre o assunto e procuramos incentivar as falas, logo a seguir afirmou:

Renata: "Porque agora a gente sabe 'né'? Antes eu não sabia fazer nada, nada. Eu nem mexia no computador quanto tinha geometria espacial."

Essas falas, ao nosso ver, mostraram que a estudante não tinha conhecimento do funcionamento do software antes das atividades do projeto de pesquisa. Além disso, o seu comportamento evidenciou como estava consciente de sua apropriação em relação às ferramentas do software e suas características: a dependência funcional geométrica, a estabilidade do objeto construído sob a ação de movimentos, e a limitação do ambiente lápis e papel em relação ao dinâmico.

A partir destas falas e do comportamento observado no vídeo, além de termos observado presencialmente suas expressões, nós verificamos a evolução naquilo que eles estavam fazendo, construindo algo novo a partir do que eles já sabiam, tendo consciência da sua evolução.

\subsection{A tarefa e o comportamento à luz do quadro teórico}

No momento em que elaboramos as tarefas, seguimos uma ordem crescente em termos de conceitos matemáticos e utilização de ferramentas do software, ponderando que os elementos técnicos e conceituais evoluem simultaneamente (BRETSCHER, 2009), buscando desafiar os estudantes. Além disso, as tarefas foram organizadas buscando extrair o máximo de informações sobre a aprendizagem do estudante nestes critérios. Quando o estudante foi submetido às tarefas, e, neste contexto, à quinta tarefa, observamos se ele construiu seguindo uma ordem lógica de inserção de objetos matemáticos procurando manter a dependência funcional geométrica. Ao realizar esta inserção mantendo a dependência funcional, consideramos que ele estivesse criando esquemas mentais (VERGNAUD, 1996), apoiando-se em seus conhecimentos prévios, 
tanto das relações matemáticas quanto das ferramentas do GeoGebra 3D. Nesta direção consideramos as palavras de Jupri, Drijvers e Heuvel-Panhuizen (2016, p. 66), os quais apontam que os "esquemas e técnicas compartilham elementos conceituais e técnicos e ambos envolvem o uso de um artefato para resolver um tipo específico de tarefa." Como os autores esclarecem, existe uma diferença importante entre os dois, sendo que os esquemas são invisíveis, entretanto, podemos observar as técnicas por meio do comportamento do sujeito enquanto ele está utilizando o artefato. Seguindo essa ideia, observamos os comportamentos dos estudantes enquanto eles estavam respondendo a quinta tarefa utilizando o GeoGebra 3D. A partir da análise dos protocolos de construções verificamos que ambos os estudantes construíram um applet o qual tinha a dependência funcional na geometria mantendo a estabilidade sob a ação de movimentos: um applet que simulou o enchimento de um reservatório cilíndrico (Figura 3 e 4).

A partir destas análises, à luz dos fundamentos já descritos, consideramos que ambos os estudantes se apropriaram do princípio da geometria dinâmica e, por consequência, ponderamos que os elementos técnicos e conceituais deles (estudantes) evoluíram simultaneamente (BRETSCHER, 2009). Ao nosso ver, ocorreu a transformação progressiva do artefato em instrumento, ou seja, a gênese instrumental (TROUCHE, 2018) dos estudantes enquanto: realizavam a quinta tarefa utilizando um artefato (GeoGebra 3D); criavam esquemas mentais (com intenção e objetivo) e elaboravam estratégias (técnicas) em como desenvolver a construção do applet do cilindro.

Seguindo nosso modelo (Figura 1), buscamos evidências da abstração refletida dos estudantes em relação ao seu processo de gênese instrumental a partir da observação de seus comportamentos enquanto realizavam as tarefas, pois o mecanismo da abstração reflexionante é uma atividade mental (BECKER, 2014).

Cada estudante apresentou indícios de abstração refletida em tempos diferentes, no entanto, ambos podem ter tido este tipo de abstração na mesma oficina instrumentada, como já mencionamos anteriormente. Consideramos que Yago teve uma abstração refletida quando fez uma análise de seu comportamento frente à realização das tarefas, além de observar que o nível de dificuldade das tarefas ia aumentando a cada oficina, e que ele evoluiu naquilo que estava fazendo. Sua fala também corroborou quando ele afirmou que o "hoje" é mais fácil, quando falou para Renata que ele também sentiu que evoluiu, no sentido de crescimento tanto em relação aos conceitos matemáticos quanto à apropriação do software.

No que tange à estudante Renata, seu comportamento foi expressivo de uma abstração refletida quando ela exclamou falando como havia mudado. Admirada com o nível das tarefas que estava realizando, se comparado ao início das oficinas, mostrando como estava consciente de sua apropriação em relação do GeoGebra 3D. Nossa análise também levou em consideração as expressões faciais dos estudantes, os quais apresentaram um olhar de quem está pensando sobre suas ações, pensando sobre o fazer, coordenando as suas ações. Estas análises foram apoiadas nas palavras de Becker (2012a, p. 96): "se o resultado de uma abstração reflexionante, de qualquer nível, torna-se consciente, teremos uma abstração refletida (réflechie) (...)". Consideramos, neste contexto, que os estudantes, Yago e Renata, tiveram uma abstração refletida, reflexionante com tomada de consciência, sobre o seu processo de gênese instrumental. Como mencionado anteriormente, este processo é contínuo por meio de reflexionamentos e reflexões, constituindo-se de um dinamismo ininterrupto provocando criação de novidades ou novas construções (BECKER, 2014). 


\section{Considerações Finais}

Não consideramos aqui os outros dados produzidos pelos instrumentos da pesquisa, tais como as anotações sobre as dúvidas que eles tinham em relação aos conceitos matemáticos e às ferramentas do software, bem como as entrevistas, a escrita e a inspirada no método clínico, a qual consideramos de grande valia para a análise de dados, dentre outros. Contudo, temos consciência de que os resultados obtidos por meio destes instrumentos nos influenciaram nas análises desta tarefa em questão e do comportamento exibido pelos estudantes na quarta oficina instrumentada. Neste artigo estamos tratando de parte dos dados da pesquisa em andamento que, ao nosso ver, já apresentam fortes indícios de que estes estudantes quando submetidos às restrições do ambiente: ultrapassaram os desafios propostos; exploraram as novas possibilidades oportunizadas pelo ambiente dinâmico; e apresentaram um comportamento específico indicando a abstração refletida sobre o seu processo de gênese instrumental.

A seleção dos dois estudantes, Renata e Yago, partiu do fato de que eles apresentaram na mesma oficina instrumentada indícios da abstração refletida. Além de ter acontecido uma interação entre ambos, sendo que nesta interação eles apresentaram evidências de uma tomada de consciência sobre seu processo de gênese instrumental, enquanto presenciamos o momento deste acontecimento. Nossa postura frente ao andamento da pesquisa, em relação à proximidade dos estudantes, foi o mais distante possível a fim de evitar interferências na gênese instrumental deles.

\section{Referências}

ARTIGUE, Michèle. Learning Mathematics in a CAS Environment: The Genesis of a Reflection about Instrumentation and the Dialectics between Technical and Conceptual Work. International Journal of Computers for Mathematical Learning, [s.1.], v. 7, n. 3, p.245-274, 2002.

BECKER, Fernando. Educação e Construção do Conhecimento. 2. ed. São Paulo: Penso, 2012a.

BECKER, Fernando. Abstração pseudo-empírica e reflexionante: significado epistemológico e educacional. Schéme: Revista Eletrônica de Psicologia e Epistemologia Genética, Marília, v. 6, p.104-128, 10 dez. 2014. Disponível em: <http://www2.marilia.unesp.br/revistas/index.php/scheme/article/view/4276>. Acesso em: 03 abr. 2016.

BRETSCHER, Nicola. Dynamic Geometry Software: The Teacher's Role in Facilitating Instrumental Genesis. In: Congress of the European Society for Research in Mathematics Education, 6., 2009, Lyon. Proceedings of CERME 6. Lyon: Cerme, 2009. p. 1340 - 1348. Disponível em: <http://ife.ens-lyon.fr/publications/editionelectronique/cerme6/wg7-30-bretscher.pdf>. Acesso em: 07 jul. 2018.

DRIJVERS, Paul et al. One episode, two lenses. Educational Studies in Mathematics, [s.l.], v. 82, n. 1, p.23-49, 7 jul. 2012.

JUPRI, Al; DRIJVERS, Paul; HEUVEL-PANHUIZEN, Marja van Den. An Instrumentation Theory View on Students' Use of an Applet for Algebraic Substitution. International Journal of Technology in Mathematics Education, S.i., v. 2, n. 23, 
p.63-80, 2016. Disponível em: <https://dspace.library.uu.nl/handle/1874/334842>. Acesso em: 10 jul. 2018.

HEGEDUS, S. J.; TALL, D. O. Foundations for the future: The potential of multimodal technologies for learning mathematics. Handbook of international research in mathematics education. 3ed., p. 543-562. New York: Taylor \& Francis, 2016. Disponível em: < http://homepages.warwick.ac.uk/staff/David.Tall/pdfs/dot2016a-tallhegedus.pdf > Acesso em 23 set.2019

MASCHIETTO, Michela; TROUCHE, Luc. Mathematics learning and tools from theoretical, historical and practical points of view: the productive notion of mathematics laboratories. Zdm, [s.1.], v. 42, n. 1, p.33-47, 29 set. 2009. Springer Nature. Disponível em: <https://link.springer.com/article/10.1007\%2Fs11858-009-0215-3>. Acesso em jul.2018.

MEIER, Melissa; GRAVINA, Maria Alice. Modelagem no GeoGebra e o desenvolvimento do pensamento geométrico no Ensino Fundamental. Instituto São Paulo GeoGebra, São Paulo, v. 1, n. 1, p.1-15, 2012. Disponível em: <http://revistas.pucsp.br/IGISP/article/view/9583/7124>. Acesso em: 22 mai. 2018.

MEDEIROS, Margarete Farias; BASSO, Marcus. Incorporação da Tecnologia Digital à Prática Docente: Compreendendo a Gênese Instrumental de Licenciandos em Matemática. Revista Novas Tecnologias na Educação: RENOTE, Porto Alegre, v. 1, n. 16, p.1-10, 2018. Semestral. Disponível em:

<https://seer.ufrgs.br/renote/article/view/85995/49363>. Acesso em: 20 ago. 2018.

NOTARE, Marcia Rodrigues. Gênese Instrumental Pessoal e Conceitos Matemáticos em Processo de Criação com o GeoGebra. Revista Novas Tecnologias na Educação: RENOTE. Porto Alegre, v.15, n.2, p.1-10, 2017. Disponível em: < https://seer.ufrgs.br/renote/article/view/79238>. Acesso em: 04 mar.2018.

RABARDEL, P. Les hommes et les technologies: une approche cognitive des instruments contemporains. Paris: Armand Colin, 1995. Disponível em: < https://hal.archives-

ouvertes.fr/file/index/docid/1017462/filename/Hommes_et_technologie_Rabardel1995. pdf>. Acesso em: 24 Mar. 2017.

ROCK, Ana Isabel Sacristán. Aprender Matemáticas en la Era Digital. Avance y Perspectiva. México, Cinvestav, V.2. n.4, 2017.

PIAGET, Jean. Abstração Reflexionante: Relações Lógico-aritméticas e Ordem das Relações Espaciais. Tradução: Fernando Becker e Petronilha Beatriz Gonçalves da Silva Porto Alegre: Artmed, 1995.

TROUCHE, Luc. Instrumentation in Mathematics Education. Encyclopedia of Mathematics Education, [s.1.], p.1-9, 2018. Springer International Publishing. Disponível em: <https://link.springer.com/referenceworkentry/10.1007\%2F978-3-31977487-9_80-3>. Acesso em: 10 set. 2019. 